\title{
Optimal Storage Policies with Wind Forecast Uncertainties
}

\author{
[Extended Abstract]
}

\author{
Nicolas Gast \\ EPFL, IC/LCA2 \\ 1015 Lausanne \\ Switzerland \\ nicolas.gast@epfl.ch
}

\author{
Dan-Cristian Tomozei \\ EPFL, IC/LCA2 \\ 1015 Lausanne \\ Switzerland \\ dan-cristian.tomozei@epfl.ch
}

\author{
Jean-Yves Le Boudec \\ EPFL, IC/LCA2 \\ 1015 Lausanne \\ Switzerland \\ jean-yves.leboudec@epfl.ch
}

\begin{abstract}
The increase in penetration of wind in the current energy mix is hindered by its high volatility and poor predictability. These shortcomings lead to energy loss and increased deployment of fast ramping generation. The use of energy storage compensates to some extent these negative effects; it plays a buffer role between demand and production. We revisit a model of real storage proposed by Bejan et al.[1]. We study the impact on performance of energy conversion efficiency and of wind prediction quality. Specifically, we provide theoretical bounds on the trade-off between energy loss and fast ramping generation, which we show to be tight for large capacity of the available storage. Moreover, we develop strategies that outperform the proposed fixed level policies when evaluated on real data from the UK grid.
\end{abstract}

\section{Keywords}

Smart Grids, Wind Power, Energy Storage

\section{INTRODUCTION}

In June 2009 the "climate and energy package" was voted into European law. This package defines the so-called "2020-20 targets": a $20 \%$ cut in emissions of greenhouse gases by 2020 , compared with 1990 levels; a $20 \%$ increase in the share of renewables in the energy mix; and a $20 \%$ cut in energy consumption. The desired increase in the energy mix of renewables, characterized by high volatility (and hence relatively high forecast errors), combined with the need for in-advance scheduling of the rest of the production, entails potential energy losses as well as an increase in the use of expensive fast ramping generation.

It has been shown [5] that the undesired effects of renewables can be mitigated via use of energy storage, with a manageable increase in energy cost. From the point of view of wind producers, this cost has an impact on profits. Several authors $[2,3]$ investigate optimal energy storage strategies for revenue maximization of wind producers in the electricity

Permission to make digital or hard copies of all or part of this work for personal or classroom use is granted without fee provided that copies are not made or distributed for profit or commercial advantage and that copies bear this notice and the full citation on the first page. To copy otherwise, to republish, to post on servers or to redistribute to lists, requires prior specific permission and/or a fee.

Greenmetrics 2012 London, UK

Copyright 20XX ACM X-XXXXX-XX-X/XX/XX ...\$10.00. market. From a wider perspective, Bejan et al. [1] investigate the cost of wind volatility in the presence of storage. They explicitly decompose this cost in two components: energy loss and the necessity of fast ramping generation. In this paper we revisit their approach in a more general setting. We redefine the two metrics of interest to account for energy losses due to inefficiencies in energy storage. We develop several heuristics and compare their performance to the ones proposed by Bejan et al. using real values of aggregated demand and wind production measured in the UK over a period of almost 3 years.

Contributions and Outline. We model a system that compensates for wind volatility by a storage system with efficiency $\eta<1$ and define the two performance metrics: energy loss and fast ramping generation (Section 2). For a storage system with large capacity, we characterize the theoretically achievable performance as a function of the storage parameters and wind volatility. We show using real data that the theoretical bound is tight and that $\eta$ has a significant impact on the performance. Incidentally, we find that for wind generation capacity of $26 \mathrm{GW}$ (which corresponds to $20 \%$ penetration), a storage of $100 \mathrm{GWh}$ largely suffices to compensate forecast errors of currently deployed prediction techniques (Section 3). Finally, for various values of the storage capacity, we show that the policy [1] that aims to maintain a constant level of charge is suboptimal and propose improved heuristics (Section 4).

\section{SYSTEM MODEL}

Our starting point is the model introduced by Bejan et al. [1]. We consider slotted time $t \in \mathbb{N}$. We assume that at each time step $t$ we observe a certain power demand $D(t)$ (expressed in Watts) that needs to be satisfied during the time slot. We use both non-dispatchable and dispatchable energy sources to satisfy the demand. Non-dispatchable energy sources provide an imposed fixed power during each time slot; a fraction of this power can be discarded. On the other hand, dispatchable energy sources can be modulated at the beginning of the time slot to exactly match the demand. Specifically, we rely on:

1. Scheduled base load power production $P_{t-n}^{f}(t)$, computed $n$ steps ahead (i.e., at time $t-n$ ),

2. Non-dispatchable wind power $W(t)$,

3. Energy storage system (e.g., pumped-storage hydro),

4. Dispatchable fast ramping generation $G(t)$ (e.g., natural gas).

Demand is always satisfied using the above energy sources. 
However, we may be confronted with energy losses if the aggregate scheduled power production and available wind power are larger than the demand.

In this paper, we analyze various power scheduling policies, i.e., heuristics for computing $P_{t}^{f}(t+n)$ at time $t$, given predictions of future wind power. The two metrics of interest in our analysis are the required amount of fast ramping generation $G$ and the amount of lost energy $L$ (which may be larger than the rejected wind production).

Due to inaccurate wind prediction, at time slot $t$ the demand $D(t)$ may not match the scheduled and non-dispatchable generation $P_{t-n}^{f}(t)+W(t)$. We denote the mismatch, i.e., the additional power required to satisfy the demand, by

$$
\Delta(t):=D(t)-W(t)-P_{t-n}^{f}(t) .
$$

Note that $\Delta(t)$ may take negative values in case of overproduction, in which case we wish to store as much of the surplus power as possible. Ideally, we also wish to use the storage system to compensate positive mismatch (fast ramping generation is expensive).

The storage system has maximum capacity $B_{\max }$. We denote by $B(t) \in\left[0, B_{\max }\right]$ the storage level at the beginning of time slot $t$. During time slot $t$, the storage system can be discharged, generating an amount of energy equal to $^{1}[B(t+1)-B(t)]^{-}$, or charged with efficiency $\eta \in[0,1]$, requiring an amount of energy equal to $\frac{1}{\eta}[B(t+1)-B(t)]^{+}$. Thus, we assume that the efficiency of a charge/discharge cycle is $\eta$. Moreover, the total amount of energy transfer is bounded by the ramping constraints: during a single time slot no more than $D_{\max }$ energy can be generated, and no more than $C_{\max }$ energy can be used for storage.

We assume that the storage system is used to reduce $|\Delta(t)|$ as much as possible. Namely, the storage level evolution at the next time step is described by a function of the current storage level and mismatch $\phi:\left[0, B_{\max }\right] \times \mathbb{R} \rightarrow\left[0, B_{\max }\right]$, that takes into account the constraints described above:

$$
\begin{aligned}
B(t+1) & =\phi(B(t), \Delta(t)), \\
\phi(B, \Delta) & = \begin{cases}\left(B-\min \left(\Delta^{+}, D_{\max }\right)\right)^{+} & \text {if } \Delta \geq 0, \\
\min \left(B+\eta \min \left(\Delta^{-}, C_{\max }\right), B_{\max }\right) & \text { if } \Delta<0 .\end{cases}
\end{aligned}
$$

If $\Delta(t) \geq 0$, then the scheduled power is insufficient. We compensate by using the storage (2) and potentially fast ramping generation for the remaining unmatched demand:

$$
G(t)=\Delta^{+}(t)-(B(t+1)-B(t))^{-} .
$$

In this case, the energy loss $L(t)$ during time slot $t$ equals 0 .

If $\Delta(t)<0$, then the scheduled production exceeds current demand, and we store the surplus (2). Naturally, there is no need for fast ramping generation, hence $G(t)=0$. The total energy loss $L(t)$ equals

$$
L(t)=\Delta^{-}(t)-(B(t+1)-B(t))^{+} .
$$

Note that as opposed to [1], the energy loss is composed of both the energy that cannot be stored and the losses due to inefficiency of the storage.

Throughout the paper, we will focus on these two metrics, both expressed in percentage of total wind energy produced:

- Fast-ramping generation: $\sum_{t} G(t) / \sum_{t} W_{t}$.

- Energy loss: $\sum_{t} L(t) / \sum_{t} W(t)$.

${ }^{1}$ Throughout the paper, we use the standard notations: $(a)^{+}=\max (a, 0)$ and $(a)^{-}=\max (-a, 0)$.
We have described the storage mechanism (2), as well as the two metrics of interest. Let us now describe the control in the form of the scheduled power $P_{t-n}^{f}(t)$.

\subsection{Scheduling policies}

In order to better cope with wind power volatility, at time slot $t$ we are given a forecast $\left\{W_{t}^{f}(t+i)\right\}_{i=1, \ldots, n}$ for the wind production at times $t+1$ to $t+n$. We assume [1] that demand is completely predictable, and thus that $D_{t}^{f}(t+$ $n) \equiv D(t+n)$. We denote by $\mathcal{F}_{t}$ the filtration at time $t$. The "base" scheduled power at time $t$ is equal to the difference in forecast demand and forecast wind production. A specific scheduling policy defines a method of computing an additional scheduled power $u_{t}^{f}(t+n)$, called the reserve. Specifically, scheduled power production $P_{t}^{f}(t+n)$ at time $t+n$ takes the following value:

$$
P_{t}^{f}(t+n):=\left(D_{t}^{f}(t+n)-W_{t}^{f}(t+n)+u_{t}^{f}(t+n)\right)^{+} .
$$

A negative value of $u^{f}$ essentially entails dispatching generation at time $t+n$ from the storage, or in the worst case from the fast ramping generation.

Let us define the three policies considered in this paper.

- At time $t$, the Fixed Reserve Policy $\mathrm{FR}(x)$ schedules the forecast mismatch at time $t+n$ between demand and wind adjusted by a constant power $u_{t}^{\mathrm{FR}}(t+n)=x$, namely

$$
P_{t}^{\mathrm{FR}}(t+n):=D(t+n)-W_{t}^{f}(t+n)+x .
$$

- We recall the Bejan-Gibbens-Kelly Policy BGK $(\lambda)$ introduced by Bejan et al. [1], which aims at maintaining a constant level of the storage $\bar{B}=\lambda B_{\max }$ (typically, $\lambda=$ $0.5)$. In order to compute $P_{t}^{\mathrm{BGK}}(t+n)$ at time $t$, this policy forecasts the storage level at time $t+n$ by using all the previously computed decisions $P_{t-n}^{\mathrm{BGK}}(t), \ldots, P_{t-1}^{\mathrm{BGK}}(t-n+1)$ and wind forecast available at time $t$ :

$$
B_{t}^{f}(t+1):=B(t+1),
$$

$B_{t}^{f}(t+i+1):=\phi\left(B_{t}^{f}(t+i), D(t+i)-P_{t+i-n}^{\mathrm{BGK}}(t+i)-W_{t}^{f}(t+i)\right)$.

Subsequently, $u_{t}^{\mathrm{BGK}}(t+n)$ is computed as the required energy to bring the storage level at time $t+n+1$ closest to $\bar{B}$ under the operating constraints:

$$
\begin{aligned}
& P_{t}^{\mathrm{BGK}}(t+n):=D(t+n)-W_{t}^{f}(t+n)+f_{\lambda}\left(B_{t}^{f}(t+n)\right), \\
& \text { where } f_{\lambda}(B)=\min \left(\frac{1}{\eta}\left(\lambda B_{\max }-B\right)^{+}, C_{\max }\right) \\
& -\min \left(\left(\lambda B_{\max }-B\right)^{-}, D_{\max }\right) \text {. }
\end{aligned}
$$

- Finally, we define the Dynamic Reserve Policy DR $(\gamma)$, for some positive value $\gamma$ that characterizes the desired tradeoff between total energy loss and total fast ramping generation. Instead of having a constant control, like the FR policy, we take the approach of Bejan et al. Namely, at time $t$ we forecast the level of the storage $B_{t}^{f}(t+n)$ at $t+n$ using previously computed control decisions via (6). We take decision $u_{t}^{\mathrm{DR}}(t+n)$ as a deterministic precomputed function $\delta_{\gamma}:\left[0, B_{\max }\right] \rightarrow \mathbb{R}$ of the forecast storage level $B_{t}^{f}(t+n)$ :

$$
P_{t}^{\mathrm{DR}}(t+n):=D(t+n)-W_{t}^{f}(t+n)+\delta_{\gamma}\left(B_{t}^{f}(t+n)\right),
$$

We characterize this function in Section 4.1.

\subsection{Demand, wind data, and forecasting}

We use data obtained from the BMRA data archive, available online at https://www.elexonportal.co.uk/. This 


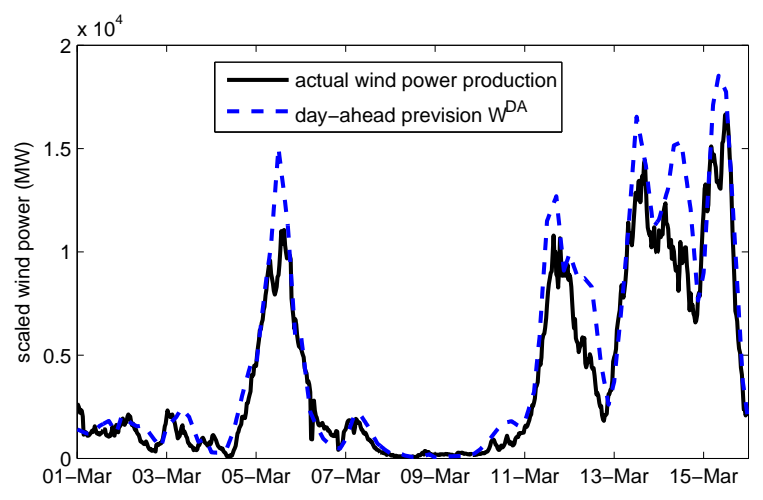

Figure 1: Typical sample of day-ahead forecast versus actual wind power production (March 2010).

archive is composed of daily reports that contain, among other things, values of aggregated electricity production and consumption in the UK.

We consider available values of aggregated demand, wind production and day-ahead wind production forecast in the time interval from June 2009 to April 2012. In the considered time frame the maximum capacity for wind power generation increased due to deployment of additional wind farms. In our analysis we consider a fixed maximum wind capacity, hence we use normalized values:

$$
W(t):=\frac{\operatorname{production}(t)}{\text { total wind capacity at time } t} \times 26 \mathrm{GW} .
$$

The value $26 \mathrm{GW}$ corresponds to $20 \%$ of wind penetration (i.e., on average $20 \%$ of the total demand can be satisfied using wind power). The considered scaling entails that during the nearly 3 years data, with the exception of a single interval of 30 minutes, the demand $D(t)$ always exceeds the wind production $W(t)$.

Every day at 3 p.m. the wind forecast is made available for the 24-hour interval starting at 9 p.m. We scale these values in the same way as the wind power (7). We concatenate them to obtain a time series of forecasts which we denote by $\hat{W}(t)$. Thus, for all time slots $t$, the forecast value $\hat{W}(t)$ was computed between 6 and 30 hours in advance.

We plot a typical sample of both scaled wind production and day-ahead forecast in Figure 1.

Based on the available forecast we employ in our analysis three ways of defining the values $\left\{W_{t}^{f}(t+i)\right\}_{i}$ that are used in the power scheduling policies:

- A first definition is the one used by Bejan et al. [1], No Prediction (NP): $W_{t}^{\mathrm{NP}}(t+i):=W(t)$. We use the current value of the wind power to predict any value in the future.

- A second definition uses the Day-Ahead (DA) prediction: $W_{t}^{\mathrm{DA}}(t+i):=\hat{W}(t+i)$. These values are well defined, as we consider $1 \leq i \leq n$, with $n$ corresponding to 6 hours.

- Finally, a third definition takes into account the fact that the process $\hat{W}(t)$ is positively correlated (as can be observed in Figure 1). We define the Corrected (cor) prediction as follows: $W_{t}^{\text {cor }}(t+i):=\hat{W}(t+i)-(\hat{W}(t)-W(t))$. Namely, we subtract the current prediction error from the day-ahead forecast for time $t+i$.

\begin{tabular}{|c|l|l|}
\hline No prediction & $W_{t}^{\mathrm{NP}}(t+i):=W(t)$ & $28 \%$ \\
\hline Day-ahead & $W_{t}^{\mathrm{DA}}(t+i):=\hat{W}(t+i)$ & $24 \%$ \\
\hline Corrected & $W_{t}^{\text {cor }}(t+i):=\hat{W}(t+i)+W(t)-\hat{W}(t)$ & $19 \%$ \\
\hline
\end{tabular}

Table 1: Wind predictions and their relative error.

The definition of the various predictions are reported on Table 1 as well as their relative error:

$$
\frac{\sum_{t}\left|W_{t}^{f}(t+n)-W(t+n)\right|}{\sum_{t} W(t)} .
$$

For this metric, $W^{\mathrm{NP}}$ is the worst forecast and $W^{\text {cor }}$ the best.

\section{LARGE SIZE STORAGE}

In this section, we obtain a lower bound on the performance of an optimal storage policy under technical conditions on the error statistics. When the storage is large, this bound can be attained by a constant reserve policy. We study the validity of this assumption on real traces in $\S 3.3$.

\subsection{A lower bound on the energy cost}

The quantity $P_{t}^{f}(t+n)$ of energy production scheduled for time $t+n$ is set at time $t$, knowing the information available at time $t$. Let $\mathcal{F}_{t}$ denote the filtration associated with the process, i.e. $\mathcal{F}_{t}$ represents the knowledge of wind production and prediction up to time $t$ but also production scheduled for time $t$ to $t+n-1$ and prediction for time $t$ to $t+n$.

The key parameter that drives the performance of the system is the error between the generation of wind energy at time $t+n$ and the forecast known at time $t$. Let $e(t+n)=$ $W(t+n)-W_{t}^{f}(t+n)$ denote this error. The next theorem states a lower bound on the performance of the system assuming that the prediction cannot be improved using the knowledge up to time $t$ and is stationary. By that, we mean that there exists a random variable $\varepsilon$ such that $e(t+n)$ is independent of $\mathcal{F}_{t}$ and distributed as $\varepsilon$. Note that we do not assume $e(t+n)$ to be independent of $\mathcal{F}_{t+1}$ to $\mathcal{F}_{t+n-1}$.

THEOREM 1. Let us assume that for all t, the forecast error $e(t+n)$ is independent of $\mathcal{F}_{t}$ and distributed as a random variable $\varepsilon$. Then, for any admissible control $u$, we have:

$$
\begin{aligned}
\bar{G} & \geq g(\bar{u}):=\mathbb{E}\left((\varepsilon+\bar{u})^{-}\right)-\operatorname{ramp}(\bar{u}) \\
\bar{L} \geq \ell(\bar{u}) & :=\mathbb{E}\left((\varepsilon+\bar{u})^{+}\right)-\operatorname{ramp}(\bar{u})
\end{aligned}
$$

where $\operatorname{ramp}(\bar{u}):=\mathbb{E}\left(\min \left(\eta(\varepsilon+\bar{u})^{+}, \eta C_{\max },(\varepsilon+\bar{u})^{-}, D_{\max }\right)\right)$ and $\bar{G}, \bar{L}$ and $\bar{u}$ are the expected time average values of $G$, $L$ and $u$, e.g. $\bar{G}=\lim _{T \rightarrow \infty} \sum_{t=1}^{T} \mathbb{E}(G(t)) / T$.

Moreover, the curve defined by $\bar{u} \mapsto(g(\bar{u}), \ell(\bar{u}))$ is convex.

\subsection{The fixed reserve policy}

Let us recall that the fixed reserve policy is a control that sets the reserve $u(t)$ to a fixed value $\bar{u}$. Theorem 1 suggests that when the storage size is large, the lower bound given by Eq. $(8,9)$ can be achieved by using a fixed reserve $\bar{u}$.

This fact is illustrated on Figure 2(a) where we consider a purely artificial scenario where the assumptions of Theorem 1 apply strictly. Namely, we explicitly add a white noise $(\varepsilon(t+n))$ to the real wind data $(W(t))$ : we consider that the wind forecast at time $t$ for time $t+i$ is

$$
W_{t}^{\mathrm{WN}}(t+i)=W(t+i)+\varepsilon(t+i),
$$




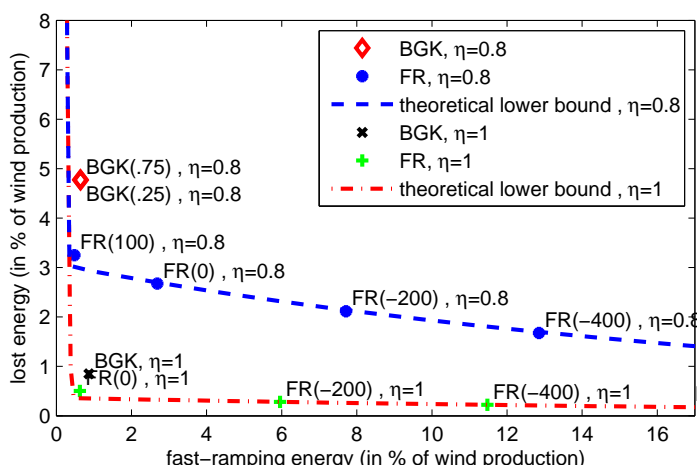

(a) Values for the white noise prediction $W^{\mathrm{WN}}$.

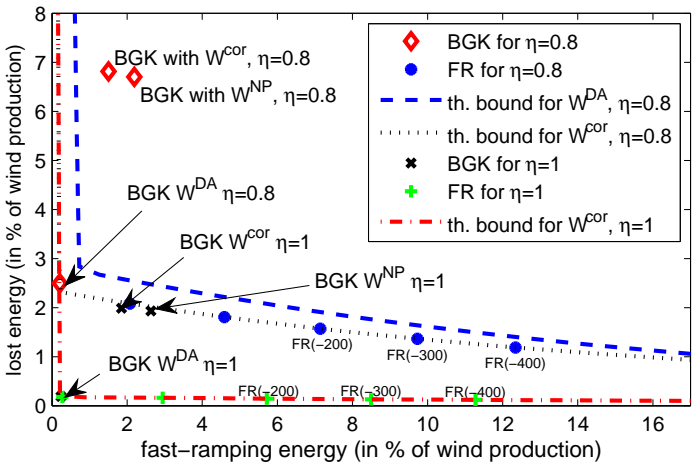

(b) Values for prediction $W^{\mathrm{DA}}$ and $W^{\text {cor }}$.

Figure 2: Performance of the fixed reserve policy, the lower bound of Theorem 1 and BGK for a large storage size $B_{\max }=100 \mathrm{GWh}$ and $C_{\max }=D_{\max }=6 \mathrm{GW}$. We compare two values of efficiency: $\eta=0.8$ and $\eta=1$.

where $\varepsilon$ is an i.i.d. sequence of Laplace random variables of mean zero and same $L^{1}$ norm as $W^{\mathrm{DA}}: \mathbb{E}(|\varepsilon|) / \mathbb{E}(W)=$ $24 \%$. Moreover, we consider a large storage system of capacity 100GWh and ramping constraints $C_{\max }=D_{\max }=$ $6 \mathrm{GW}$, in order to avoid energy losses due to overflow.

In Figure 2(a) we plot with dashed lines the parametric curve defined in Theorem 1 for two values of the efficiency: $\eta=0.8$ and $\eta=1$. We also plot the performance of the fixed reserve policy $\mathrm{FR}(\delta)$ for various values of the reserve $\delta$, as well as the BGK threshold policy for two values of the thresholds 0.25 and 0.5 .

There are two important observations. First, the fixed threshold policy always achieves the lower bound. Second, whereas when $\eta=1$ the performance of all policies is similar, when $\eta<1$, the BGK policy is far from being optimal and looses $50 \%$ more energy than the best fixed reserve policy.

\subsection{Real data prediction values}

We apply the same method to compare the performance of fixed reserve policies against BGK on real forecast data. We compare the performance of BGK for the three wind forecasts $W^{\mathrm{NP}}, W^{\mathrm{DA}}$, and $W^{\text {cor }}$ against the performance of the fixed reserve policy for the corrected forecast $W^{\text {cor }}$.

In Figure 2(b) we plot the curve defined in Theorem 1 using the measured errors for the day-ahead forecast $W^{\mathrm{DA}}$ (blue dashed line) and the corrected forecast $W^{\text {cor }}$ (black dotted line for $\eta=0.8$, red dashed-dotted line for $\eta=1$ ). Note that, since the hypothesis on the forecast error is not respected, the Theorem does not give any guarantees.

For both $\eta=0.8$ and $\eta=1$, the performance of the BGK policy run with $W^{\mathrm{DA}}$ is very close to the lower bound obtained for the corrected forecast. However, its performance decreases when run with the (theoretically) more accurate prediction $W^{\text {cor }}$. We attribute this fact to the positive correlations between the the errors $W^{\mathrm{DA}}$ that become negative correlations for $W^{\text {cor }}$

\section{INFLUENCE OF STORAGE SIZE}

The theoretical lower bound depends only on the efficiency $\eta$ and the ramping constraints $C_{\max }$ and $D_{\max }$, but not on the total storage capacity $B_{\max }$. The previous section shows that for a storage system of $100 \mathrm{GWh}$ the performance of the fixed reserve policy is very close to this lower bound. In this section, we analyze performance for a smaller storage capacity $^{2}$, ranging from $5 \mathrm{GWh}$ to $50 \mathrm{GWh}$.

\subsection{The dynamic reserve policy}

In this section, we fully describe the family of dynamic reserve policies defined in $\S 2.1$. These policies take into account the state of the storage to decide the value of the reserve. Each policy is derived from the optimal policy of a Markov Decision Process (MDP) that represents a simplified model. The state space of the MDP is $\left[0, B_{\max }\right]$, and the action space is $\mathbb{R}^{+}$. If $X(t)$ is the state at time $t$ and $u(t)$ the control taken at time $t$, the state at time $t+1$ is:

$$
X(t+1)=\phi(X(t),-\varepsilon(t+1)-u(t)),
$$

where $\varepsilon$ is an i.i.d. sequence of variables on $\mathbb{R}$, with the same distribution as the error of the corrected forecast $W(t+n)-$ $W_{t}^{\text {cor }}(t+n)$. The function $\phi$ represents the evolution of the storage in one time step and is defined by Eq.(2). Given a state $x$ and an action $u$, the instantaneous cost is a weighted sum of the lost energy and the fast ramping generation:

$$
\begin{aligned}
c(X, u)= & \mathbb{E}\left((\varepsilon+u+X-\phi(X,-\varepsilon-u)) \mathbb{1}_{\varepsilon+u>0}\right) \\
& +\gamma \mathbb{E}\left((\phi(X,-\varepsilon-u)-X-\varepsilon-u) \mathbb{1}_{\varepsilon+u<0}\right) .
\end{aligned}
$$

Eq.(11) is a cost corresponding to the expected lost energy (derived via Eq.(4)), Eq.(12) corresponds to the fast ramping generation (derived via Eq.(3)). The weight $\gamma$ characterizes the trade-off and indexes the family of policies.

We seek to minimize the infinite horizon average cost:

$$
v^{*}:=\min _{u(.)}\left\{\lim _{T \rightarrow \infty} \frac{1}{T} \sum_{t=1}^{T} \mathbb{E}(c(X(t), u(t)))\right\} .
$$

There exists a function $v:\left[0, B_{\max }\right] \rightarrow \mathbb{R}$, unique up to an additive constant that satisfies Bellman's equation [4]:

$$
v(x)=\min _{u \in \mathbb{R}} \mathbb{E}(c(x, u)+v(\phi(x,-u-\varepsilon)))-v^{*} .
$$

We compute $v$ of Eq.(13) by discretizing the state space and the action space, through application of the value iteration

\footnotetext{
${ }^{2}$ To give an order of magnitude, the Dinorwig power station in Wales cited by Bejan et al. [1] has a capacity of $B_{\max }=$ $9 \mathrm{GWh}$ and $D_{\max }=1.8 \mathrm{GW}$ and has an efficiency $\eta=0.75$. http://www.fhc.co.uk/dinorwig.htm
} 


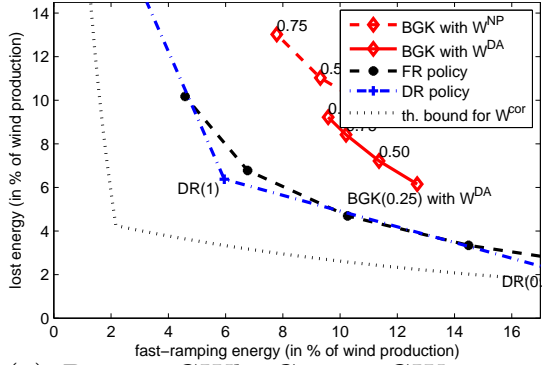

(a) $B_{\max }=5 \mathrm{GWh}, C_{\max }=2 \mathrm{GW}, \eta=0.8$

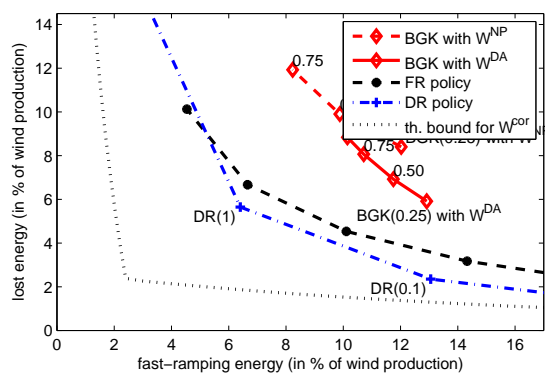

(d) $B_{\max }=5 \mathrm{GWh}, C_{\max }=2 \mathrm{GW}, \eta=1$

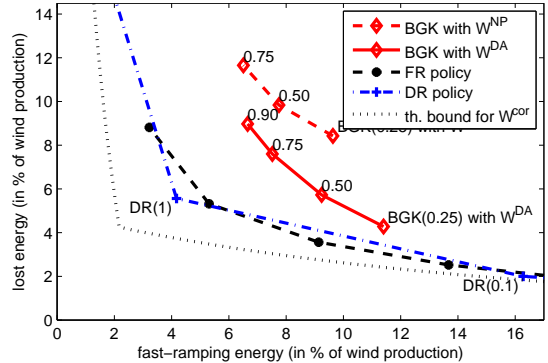

(b) $B_{\max }=10 \mathrm{GWh}, C_{\max }=2 \mathrm{GW}, \eta=0.8$

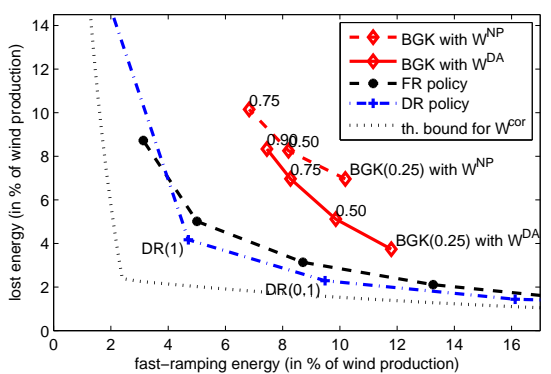

(e) $B_{\max }=10 \mathrm{GWh}, C_{\max }=2 \mathrm{GW}, \eta=1$

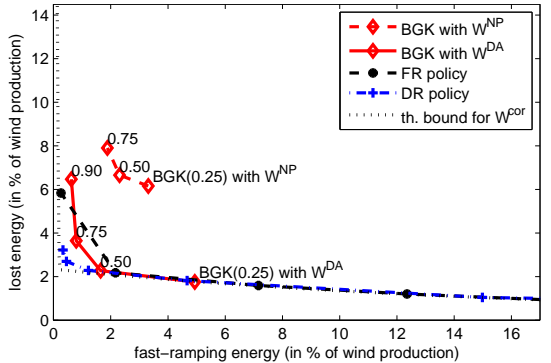

(c) $B_{\max }=50 \mathrm{GWh}, C_{\max }=6 \mathrm{GW}, \eta=0.8$

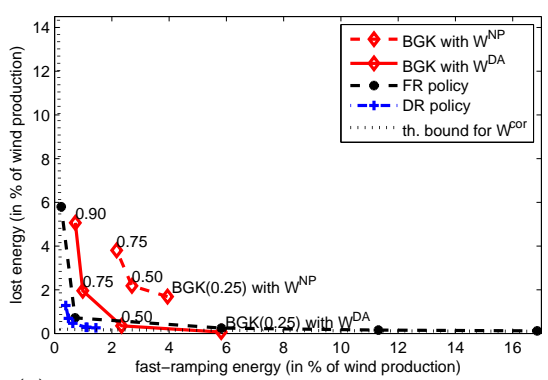

(f) $B_{\max }=50 \mathrm{GWh}, C_{\max }=6 \mathrm{GW}, \eta=1$

Figure 3: Performance of the fixed reserve policy, the BGK policy and the dynamic reserve policy for various storage capacity and ramp constraints. The top line (Figures $(\mathbf{a}, \mathbf{b}, \mathbf{c})$ ) are for an efficiency of $\eta=0.8$ while the bottom line (Figures $(\mathbf{d}, \mathbf{e}, \mathbf{f}))$ is for $\eta=1$.

method. From $v$, we derive an optimal policy $\delta_{\gamma}$ that depends on the ratio $\gamma$ :

$$
\delta_{\gamma}(x):=\underset{u \in \mathbb{R}}{\arg \min }\{\mathbb{E}(c(x, u)+v(\phi(x,-u-\varepsilon)))\} .
$$

For each value $\gamma$, we obtain a policy for the original system as follows. From (6), we compute an estimation of the state of the storage at time $t+n, B_{t}^{f}(t+n)$. The dynamic reserve policy is defined by setting the production at time $t+n$ to:

$$
P_{t}^{\mathrm{DR}}(t+n):=D^{f}(t+n)-W_{t}^{f}(t+n)+\delta_{\gamma}\left(B_{t}^{f}(t+n)\right)
$$

\subsection{Numerical evaluation}

To evaluate the performance of the different policies, we simulate the fixed reserve policy (FR), dynamic reserve policy (DR), and the BGK policy for various sizes of the storage capacity and ramp constraints: $\left(B_{\max }, C_{\max }=D_{\max }\right)$ equal to $(5 \mathrm{GWh}, 2 \mathrm{GW}),(10 \mathrm{GWh}, 2 \mathrm{GW})$ and (50GWh, 6GW). The results are shown in Figure 3 for $\eta=0.8$ and $\eta=1$. For BGK, we plot both the values obtained with $W^{f}=W^{\mathrm{NP}}$ (the original algorithm described in [1]) and with the dayahead forecast $W^{\mathrm{DA}}$ (which gives the best performance for this policy among all considered forecasts: $W^{\mathrm{NP}}, W^{\mathrm{DA}}$, and $\left.W^{\text {cor }}\right)$. For the dynamic reserve policies, we use the corrected forecast $W^{\text {cor }}$ and we vary $\gamma$ from 0.01 to 100 .

The plots show that, for all tested values of storage size and ramp up constraints, the BGK policies with day-ahead forecast - that try to maintain the storage at a fixed level are outperformed by both the fixed reserve and the dynamic reserve policies with corrected forecast. Even when $\eta=1$, which intuitively would favor BGK, the DR policy reduces both the energy loss and the fast ramping generation. This suggests that taking into account the statistical nature of the prediction error for time $t+n$ leads to higher performance gains than trying to maintain the storage in a balanced state.

\section{CONCLUSIONS}

In this paper, we consider different policies governing a storage system to compensate for wind prediction errors. We show that incorporating an efficiency factor $\eta<1$ when charging the storage system leads to dramatically different conclusions than for $\eta=1$. We provide a lower bound on the performance that shows that when $\eta<1$, the total energy loss plus fast ramping generation used will be strictly positive, even with infinite storage size. We provide two heuristics that attain this lower bound when the storage capacity is large and that outperform existing heuristics when this capacity is low.

\section{REFERENCES}

[1] A. Bejan, R. Gibbens, and F. Kelly. Statistical aspects of storage systems modelling in energy networks. 46th Annual Conference on Information Sciences and Systems, March 2012.

[2] E. D. Castronuovo and J. A. P. Lopes. Optimal operation and hydro storage sizing of a wind-hydro power plant. International Journal of Electrical Power \& Energy Systems, 26(10):771 - 778, 2004.

[3] M. Korpaas, A. T. Holen, and R. Hildrum. Operation and sizing of energy storage for wind power plants in a market system. International Journal of Electrical Power 83 Energy Systems, 25(8):599 - 606, 2003.

[4] M. Puterman. Markov decision processes: Discrete stochastic dynamic programming. J. W. \& S., 1994.

[5] G. Strbac, A. Shakoor, M. Black, D. Pudjianto, and T. Bopp. Impact of wind generation on the operation and development of the uk electricity systems. Electric Power Systems Research, 77(9):1214 - 1227, 2007. 